\title{
Application of Polarized Light Spectroscopy to Study of Liquid Crystalline Phases
}

\author{
Danuta Bauman
}

The following optical spectroscopy methods used to study of liquid crystals have been discussed: infrared, visible and ultraviolet absorption spectroscopy, fluorescence depolarization and Raman scattering. It has been shown, that using these methods the long-range orientational order in uniaxial liquid crystalline phases as well as some direction-dependent spectroscopic molecular properties of compounds under investigation can be determined.

\section{Introduction}

Liquid crystals were discovered in 1888 by Austrian botanist Reinitzer who synthesized several esters of cholesterol and found in these esters the phenomenon of "double melting", this means at a certain temperature the compound changed from the crystalline phase to an opaque liquid which transformed at defined higher temperature to an optically clear liquid. In the opaque liquid phase the optical anisotropy was observed. This new phase was called "fluid crystals" or "liquid crystals" (fließende Kristalle, fluissige Kristalle). The structure of a liquid crystal, called also mesomorphic structure, results from anisotropic intermolecular interactions which lead to the long-range positional and/or orientational order being reason of anisotropic properties of these substances. Unique combination in the mesomorphic substances of the long-range orientation with the properties of liquid, which can be easy deformed, has caused, that in the last thirty years liquid crystalline materials have found application in the most modern fields of science and technology. Great interest for these substances is connected with the role, which they play in giving information concerning the development and excellence of the condensed matter theory. In electronic technology the liquid crystals have attracted considerable attention because of request on the substances with specific optical properties used in display devices. Liquid crystal displays are in general passive displays: they do not emit the light and can be observed in the transmissive or reflective light. This passiveness causes that their most important advantages are: low power consumption, easiness of the construction and possibility of viewing in the bright light, inclusive the direct sunlight. This allows on the display miniaturization and application in mobile devices. However the greatest advantage of TV and computer screens utilizing liquid crystals is their flatness.

In many thousands of organic compounds the liquid crystalline state appears by heating the substance above its melting temperature. It can be identified visual (we can see characteristic opaque liquid) or by means of polarizing microscope (the optical birefringence is observed). By further heating liquid crystal transforms in isotropic liquid. Compounds which exhibit the liquid crystalline state in a certain temperature interval are called "thermotropic" liquid crystals. There exist also "lyotropic" liquid crystals, which may be formed by the combined action of polar amphiphilic compounds and certain solvents, for example water. They exist in certain regions depending upon the concentration and the temperature.

The fundamental criterion, which determines, if the given substance can have the liquid crystalline phase is geometric anisotropy of molecules. On the basis of experiments, carried out over 90 years, the general rule was accepted, that the molecules of thermotropic liquid crystals must have rod-like shape. Meanwhile, in 1977 it was discovered that also compounds with disc-like molecular shape are able to form liquid crystals of different phase structures. In recent years the gap between rod-like and disc-like molecules could be filled with lathlike molecules, which are also able to form the liquid crystalline phases. So, derived from the shape of the constituting molecules, we may differentiate among [1]:

- calamitic liquid crystals occurring in rod-like molecules, which may be considered as the classical liquid crystals,

- discotic liquid crystals derived from disc-like molecules, which were discovered in 1977 ,

- sanidic liquid crystals occurring in lath-like (board-like) molecules, which were first found in 1986.

Calamitic liquid crystals are devided into nematics and smectics. In the nematic phase, as in the isotropic phase, there is a very short-range positional order in all directions, and the molecules can translate freely. But the nematic phase shows an orientational order such that the long molecular axes are overall parallel to the direction indicated by the vector $\mathrm{n}$, called "director". Both positional order and orientational order are necessary for a smectic liquid crystalline phase. There are many different types of smectic phases. According to the chronological sequence of their detection the smectic phases have been designated with code letters A, B, C, ..., M. Two important cases are when the director is parallel to the layer normal (smectic A phase) and when the director is at an angle to the layer normal (smectic $\mathrm{C}$ phase). Other smectic phases exist in which short-range positional order or bond orientational order develops within the plane of the layers. There are many examples, each with a different combination of in-plane order and the angle between the layer normal and the director. If the molecules constituting these phases are chiral, which means that they do not possess inversion symmetry, then the nematic phase and some smectic phases do not occur. Instead, they are replaced by chiral versions of 
these phases with different physical structures. For example, in a chiral nematic phase (previously called cholesteric phase) the director rotates along a direction perpendicular to the director. Similarly, in place of the smectic $C$ phase the director maintains a constant tilt angle with respect to the layer normal and rotates about a cone in going from one layer to the next.

Thermotropic liquid crystalline structures can be created not only by molecules with low molecular mass but also by polymers. For the most polymers the structural units responsible for the formation of the mesophase can be distinguished. They are called mesogenic groups. Considering the position of these groups we have mainchain polymers, side-chain polymers and polymers with mixed structure.

Between solid and isotropic states can occur one liquid crystalline phase - we say about monomorphism, two phases - dimorphism, up to six - hexamorphism. Generally - polymorphism. The polymorphism in the liquid crystal state undergoes a certain rule of the phase sequence. This rule in polymorphic compounds predicts a stepwise decrease of order with increasing temperature and the reverse with decreasing temperature. This means, according to this rule, the smectic phases generally are low-temperature phases with respect to the nematic phase.

As is known, the classical methods of molecular spectroscopy, this means optical spectroscopy with polarized light and magnetic resonance, have been widely applied to study of anisotropic systems, among others thermotropic and lyotropic liquid crystals as well as soluteliquid crystal mixtures. Linearly polarized light is of special interest because it represents a powerful tool for study of oriented or partially oriented assemblies of molecules [2].

In this review paper the following spectroscopic methods using linearly polarized light will be briefly described: infrared (IR), visible (VIS) and ultraviolet (UV) absorption spectroscopy, fluorescence depolarization (FD) and Raman scattering (RS). It will be shown, that using these methods one can obtain information about the long-range orientational order in liquid crystalline systems $[1,3,4]$. Particular point will be made about investigations of the guestliquid crystal mixtures, because they can be utilized in two ways: to determine the molecular spectroscopic properties of the guest molecules, for example the polarization of the electronic absorption and emission transitions in UV, VIS and fluorescence spectroscopy or vibrational transitions in IR and RS spectroscopy [1-3] on the one hand and to characterize the structure of the uniaxial liquid crystalline phases on the other $[1,3,4]$. In this latter case the guest molecules can be used as probes to reflect the orientational properties and dynamics of the host medium.

\section{Infrared Spectroscopy}

IR spectra of large molecules, such as a liquid crystal, are mostly very complex due to the considerable mixing of the vibrational modes in the potential energy distribution. Therefore, IR spectroscopy seems to be not all too convenient method for the study of the molecular properties of the guest dissolved in the liquid crystalline host. However, by careful selection, it is possible to determine the polarization of some vibrations of solutes. On the basis of the polarized IR spectra the order parameter $\mathrm{S}[\mathrm{u}]$ of a liquid crystal or that of a dissolved molecule can be determined. In order to make this, an appropriate absorption band should be chosen. The band must have sufficient intensity and be not overlapped by neighbouring bands. Moreover, it should belong to a non-degenerated vibration with known orientation of the vibrational transition moment with respect to the long axis of the guest or host molecule. The order parameter $S$ is then connected with the degree of anisotropy $\mathrm{R}$ via the following relation [1,3]

$$
\mathrm{S}=\frac{\mathrm{R}}{\frac{1}{2}\left(3 \cos ^{2} \vartheta-1\right)} .
$$

$\vartheta$ is here the angle between the transition moment direction and the long molecular axis, and $\mathrm{R}$ is described as follows

$$
\mathrm{R}=\frac{\varepsilon \|-\varepsilon \perp}{3 \varepsilon} \text {. }
$$

$\varepsilon_{\|}$and $\varepsilon_{\perp}$ are the molar decadic absorption coefficients for the light polarized parallel and perpendicularly to the optical axis of the liquid crystal sample and $\varepsilon$ is the absorption coefficient determined in the following way

$$
\varepsilon=\frac{1}{3}\left(\varepsilon \|+2 \varepsilon_{\perp}\right) .
$$

Using IR spectra the temperature dependence of the order parameter for some liquid crystals $[5,6]$ and some guest molecules [3] had been determined. On the other hand, knowing the order parameter, i.e. from other experiment, the value of the degree of anisotropy of a given absorption band can be used to obtain information about the polarization of the corresponding transition moment.

\section{Ultraviolet and Visible Spectroscopy}

The majority of liquid crystals used to the optical studies have very high absorption coefficient in the UV spectral region [1], and some, i.e. azoxybenzenes and Schiff bases absorb also in the near VIS region, what is due to their large conjugated $\pi$-electron systems. Solely bicyclohexanes are transparent down to ca. $200 \mathrm{~nm}$. From this reason UV-VIS spectroscopy is rather rarely used to study of the pure liquid crystals. On the contrary, this kind of spectroscopy is very often utilized to the determination of both the molecular properties as well as the order parameter of the dissolved guest molecules. Especially, the liquid crystals doped with various dichroic dyes had been object of very intensive investigations [7-11], because of their potential application in the guest-host liquid crystal displays $[11,12]$. If the appropriate liquid crystal is chosen as solvent, then not only the order parameter, but also the polarization of the absorption electronic bands can be estimated simultaneously from the measurements of the polarized UV-VIS spectra [10,13-15]. 


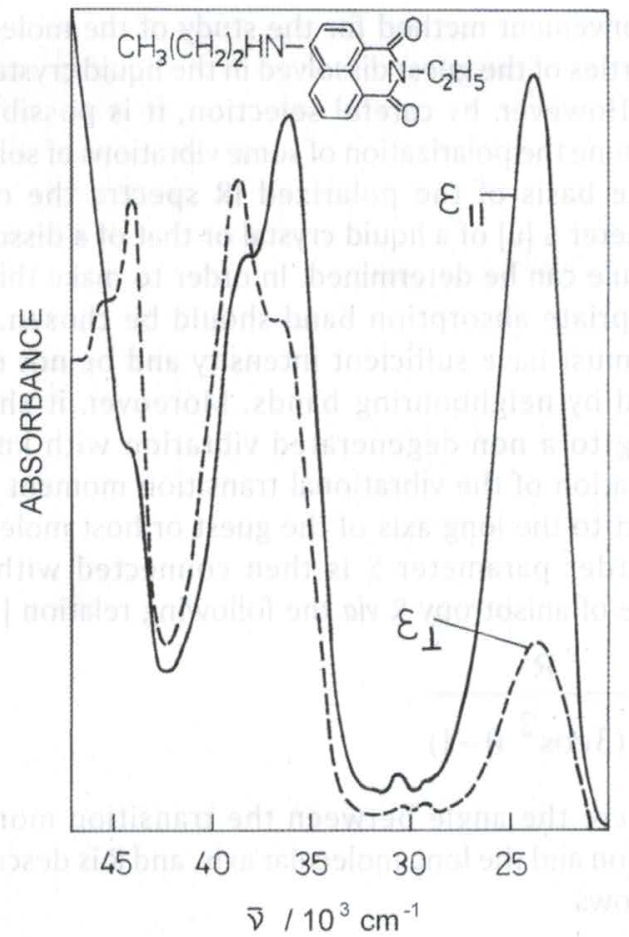

Fig. 1: Polarized absorption spectra of dichroic dye in liquid crystal ZLI 1695 at $T=300 K[13]$

Figure 1 shows the polarized components of the absorption spectra of one of naphthalene derivatives of bicarboxylic acid in liquid crystal ZLI 1695 (Merck) as an example [13]. From such spectra the degree of anisotropy $\mathrm{R}$ can be obtained in the some way as from IR spectra. $R$ is connected with the guest order parameters $S$ and $D$ by relation $[10,13-16]$

$$
R=S\left(1-\frac{3}{2} \sin ^{2} \vartheta\right)+\frac{\sqrt{3}}{2} \mathrm{D} \sin ^{2} \vartheta \cos 2 \varphi,
$$

where $\vartheta$ and $\varphi$ are the angular polar coordinates of the absorption transition moment in the molecular frame. The order parameter S characterizes the orientation of the molecular axis with respect to the optical axis of the uniaxial phase and the parameter $D$ is a measure for the deviation from a rotationally symmetrical distribution of the molecules and is often called the "biaxiality parameter".

$\mathrm{S}$ and $\mathrm{D}$ can be in principle determined from the degree of anisotropy of any two bands for which the orientations of the related electric dipole transition moments with respect to the molecular axes are different and known. For two purely polarized bands at $\bar{v}_{1}$ and $\bar{v}_{2}$

$$
\begin{aligned}
& \mathrm{S}=\mathrm{R}\left(\overline{\mathrm{v}}_{1}\right), \\
& \mathrm{D}=\frac{1}{\sqrt{3}}\left(2 \mathrm{R}\left(\overline{\mathrm{v}}_{2}\right)+\mathrm{R}\left(\overline{\mathrm{v}}_{1}\right)\right) .
\end{aligned}
$$

If there exists only one absorption region with a purely polarized band at $\bar{v}_{1}$ and another band $\left(\bar{v}_{2}\right)$ of mixed polarization, then more complicated procedure must be used [10,13-15].

The most dichroic dyes used in the liquid crystal displays, i.e. azo dyes or anthraquinone dyes, have uniaxial symmetry and only one absorption band in the VIS region, connected with one absorption transition moment, which direction is in general parallel or almost parallel to the long molecular axis of the dye $[8,9]$. Then the order parameter $\mathrm{S}$ can be easily obtained from the degree of anisotropy of this band and D is equal to zero. Figure 2 presents $\mathrm{S}$ as a function of reduced temperature $\mathrm{T}^{*}=\mathrm{T} / \mathrm{T}_{\mathrm{NI}}$ ( $\mathrm{T}_{\mathrm{NI}}$ is the nematic-isotropic phase transition temperature in K) for the liquid crystal 8CB (4-n-octyl-4'-cyanobiphenyl) doped with three azo dyes with various molecular structure [17]. For comparison, the values of $\mathrm{S}$ parameters for pure $8 \mathrm{CB}$, obtained from optical birefringence measurements are also presented in this figure. It can be seen, that the order parameter of the guest molecules can be higher or lower than that of the host molecules at the same reduced temperature. However, if the appropriate dye for given liquid crystal is chosen, then very good correlation of the orientation between guest and host molecules can occur (i.e. for dye 1 and 8CB in Fig. 2). In such cases it is possible to obtain information about the host orientation on the basis of the polarized absorption spectra of the guest oriented in the liquid crystalline solvent. This is a very simple method of the determination of the orientational order in the uniaxial liquid crystalline phases.

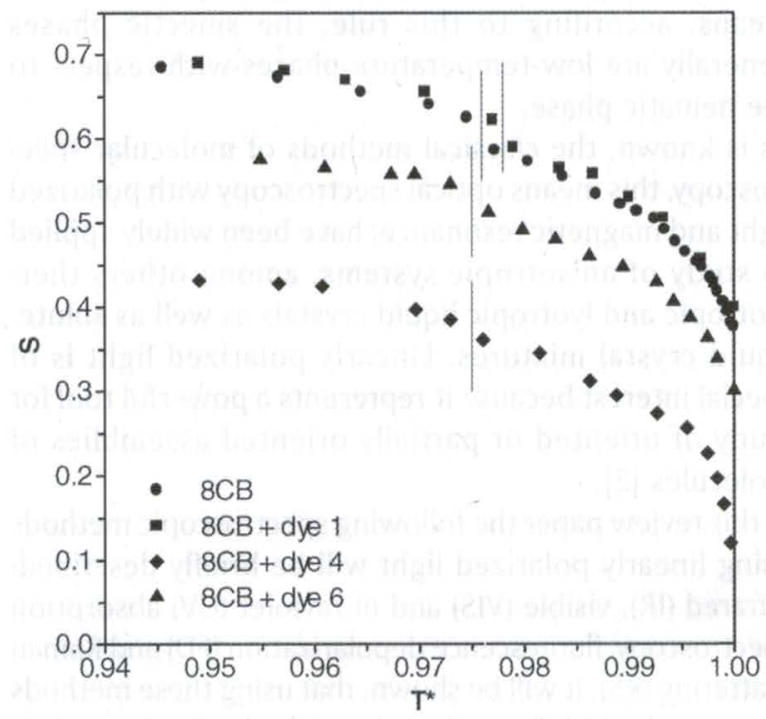

Fig. 2: Temperature dependence of the order parameter $S$ for pure $8 C B$ and for dyes dissolved in $8 C B$. Bars indicate the temperature of the smectic $A-$ nematic transition [17].

\section{Fluorescence Depolarization}

Polarized fluorescence spectra of the guest molecules can be measured in the liquid crystal solvents in the same way as polarized absorption spectra. Again, the different intensity of the fluorescence with light emitted parallel and perpendicularly to the liquid crystal optical axis can be used to determine the polarization of the fluorescence bands of the dichroic guest molecules [18]. Moreover, the fluorescence depolarization is one of the optical spectroscopic methods allowing to estimate simultaneously the order parameters $\left\langle\mathrm{P}_{2}\right\rangle$ and $\left\langle\mathrm{P}_{4}\right\rangle$ [4] from one experiment. The theory of time dependent fluorescence depolarization in liquid crystals and its application to the evaluation of the orientational order had been first given by Zannoni [19]. Four components of the polarized fluorescence spectra of the fluorescent 
probe molecule dissolved in a liquid crystalline host can be measured in various geometries, which allow to determine two emission anisotropies

$R_{1}=\frac{J_{z z}-J_{z y}}{J_{z z}+2 J_{z y}}, R_{2}=\frac{J_{y z}-J_{y y}}{J_{y z}+2 J_{y y}}$,

where the first subscript denotes the direction of the incident light and second refers to the direction of the emitted light in the laboratory frame $x, y, z$. Jij $_{\text {ij }}$ 's are reduced fluorescence intensities, regarding the corrections for instrumental, concentration and volume factors.

For the angle $\vartheta=0^{\circ}$ and assuming that the lifetime of the fluorescent molecule $\tau_{\mathrm{F}}$ is much shorter than its relaxation time $\tau_{R}$, the following relations between the emission anisotropies and the order parameters can be obtained

$$
\begin{aligned}
& \mathrm{R}_{1}=\frac{\frac{2}{5}+\frac{11}{7}<\mathrm{P}_{2}>+\frac{36}{35}<\mathrm{P}_{4}>\mathrm{P}_{2}(\cos \delta)}{1+2<\mathrm{P}_{2}>}, \\
& \mathrm{R}_{2}=\frac{\left[<\mathrm{P}_{2}>-\frac{2}{5}-\frac{11}{35}<\mathrm{P}_{4}>\right] \mathrm{P}_{2}(\cos \delta)}{1-\frac{1}{3}<\mathrm{P}_{2}>+2\left[\frac{1}{5}-\frac{2}{7}<\mathrm{P}_{2}>+\frac{3}{35}<\mathrm{P}_{4}>\mathrm{P}_{2}(\cos \delta)\right.} .
\end{aligned}
$$

$\delta$ is here the intramolecular angle between absorption and emission oscillators and $\left\langle\mathrm{P}_{2}\right\rangle$ and $\left\langle\mathrm{P}_{4}\right\rangle$ are the order parameters, as defined in [u].

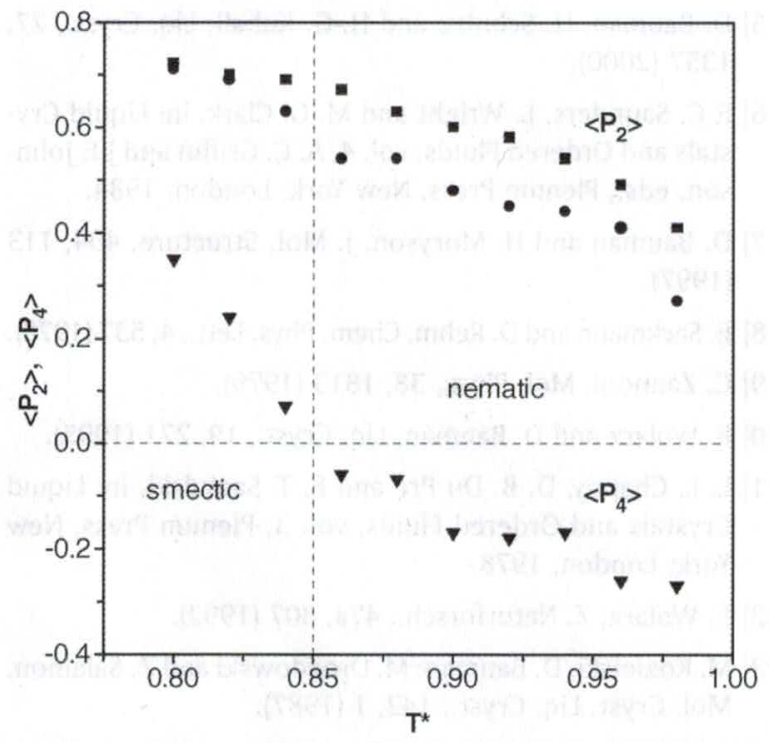

Fig. 3: Order parameters $\left\langle P_{2}>\right.$ and $\left\langle P_{4}\right\rangle$ versus reduced temperature for liquid crystalline polysiloxane doped with DANS: squares $-\left\langle P_{2}\right\rangle$ determined from absorption, circles $-\left\langle P_{2}\right\rangle$ and triangles $-\left\langle P_{4}\right\rangle$ determined from fluorescence measurements [20].

The assumption that $\tau_{R}>\tau_{F}$ is reasonable for very viscous substances, e.g. low molecular liquid crystals in smectic phases and liquid crystalline polymers. Figure 3 shows the temperature dependence of the order parameters $\left\langle\mathrm{P}_{2}\right\rangle$ and $\left\langle\mathrm{P}_{4}\right\rangle$ obtained on the basis of the fluorescence measurement of dimethylamino-nitrostilbene (DANS) in one of polysiloxanes with side mesogenic groups [20]. For DANS molecule the angle $\vartheta$ is equal to $0^{\circ}$, and $\delta$ had been calculated to be $(13 \pm 4)^{\circ}$ [20]. For comparison, in this figure the order parameter $\left\langle\mathrm{P}_{2}\right\rangle$ obtained from the polarized absorption spectra is also presented. The negative values of $\left\langle\mathrm{P}_{4}\right\rangle$ in the nematic phase indicate the strong tendency of the mesogenic groups in the less viscous nematic phase to tip away from the macroscopic orientation direction, which is probably connected with the perturbation of the uniform molecular arrangement of the side chains by the rigid polymer backbone.

More complicated expressions allowing to calculate $\left\langle\mathrm{P}_{2}\right\rangle$ and $\left\langle\mathrm{P}_{4}\right\rangle$ for the case when $\vartheta \neq 0^{\circ}$ and $\tau_{\mathrm{F}} \approx \tau_{\mathrm{R}}$ can be found in literature $[19,21,22]$.

\section{Raman Scattering}

Raman scattering, like as fluorescence, is a two-photon process, but the difference in the time scale occurs. While the lifetime of the molecule in the excited state is of order of $10^{-7}-10^{-9} \mathrm{~s}$, the Raman scattering process takes place almost immediately after absorption. Therefore, in order to use the RS spectroscopy to study of the orientational order of liquid crystals, the relaxation time of molecules does not need to be taken into account. Otherwise, the method allowing to determine the order parameters $\left\langle\mathrm{P}_{2}\right\rangle$ and $\left\langle\mathrm{P}_{4}\right\rangle$ is quite similar to that

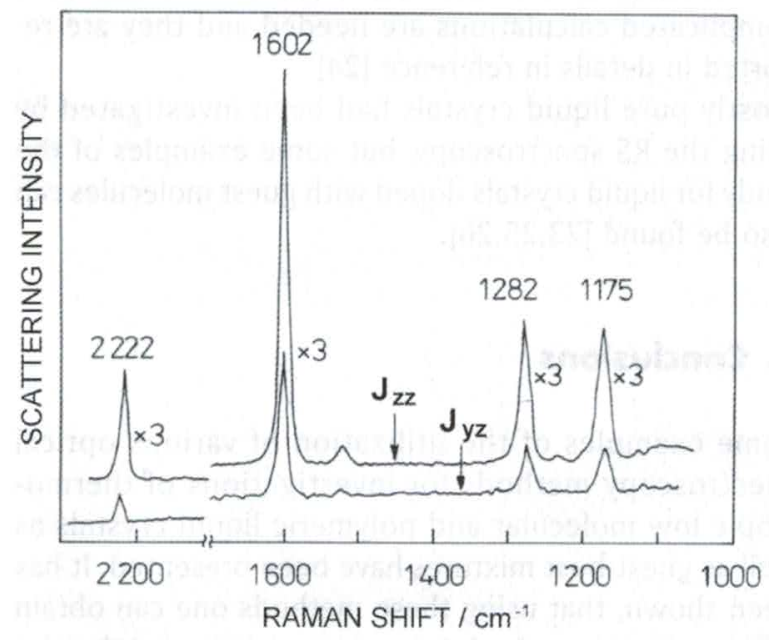

Fig. 4: Polarized Raman scattering spectra $\left(J_{z z}, J_{y z}\right)$ of $5 C B$ at $T=300 K$ [23].

utilizing FD.

Figure 4 presents the polarized components of the RS spectra for the liquid crystal 5CB (4-n-pentyl-4'-cyanobiphenyl) [23]. The band intensities of $J_{z z}$ component are always significantly larger than those of $\mathrm{J}_{\mathrm{yz}}$, which indicates that RS band intensities vary with the molecular orientation and therefore they can reflect the molecular order of liquid crystal in thin oriented layer. Measuring the intensity of the scattered light in various geometries it is possible to evaluate three depolarization ratios

$\mathrm{r}_{1}=\frac{\mathrm{J}_{\mathrm{zy}}}{\mathrm{J}_{\mathrm{zz}}}, \mathrm{r}_{2}=\frac{\mathrm{J}_{\mathrm{yz}}}{\mathrm{J}_{\mathrm{yy}}}, \mathrm{r}_{3}=\frac{\mathrm{J}_{\mathrm{xy}}}{\mathrm{J}_{\mathrm{xx}}}$,

where the first subscript denotes now the polarization of the incident light and the second refers to that of the scattered light in the laboratory frame $x, y, z$. $\mathrm{J}_{\mathrm{ij}}$ 's must require corrections for the anisotropy of the refractive index and of the apparatus polarization. 
It is always possible to find the particular vibration, for which the polarizability tensor related to the molecular frame has the diagonal form [24]

$\hat{\alpha}=\left(\begin{array}{lll}a & 0 & 0 \\ 0 & b & 0 \\ 0 & 0 & 1\end{array}\right)$

For the uniaxial liquid crystal molecules it is reasonable to assume that $\mathrm{a}=\mathrm{b}$. This value can be easily determined on the basis of the depolarization ratio in the isotropic phase [24]. If $\mathrm{a} \approx \mathrm{b} \cong 0$ or in the case of resonance Raman technique the order parameters $\left\langle\mathrm{P}_{2}\right\rangle$ and $\left\langle\mathrm{P}_{4}\right\rangle$ can be calculated on the basis of two depolarization ratios $r_{1}$ and $r_{2}$ only

$$
\begin{aligned}
& <\mathrm{P}_{2}>=\frac{3 \mathrm{r}_{1} \mathrm{r}_{2}-4 \mathrm{r}_{1}+3 \mathrm{r}_{2}}{12 \mathrm{r}_{1} \mathrm{r}_{2}+8 \mathrm{r}_{1}+3 \mathrm{r}_{2}}, \\
& <\mathrm{P}_{4}>=\frac{3\left(\mathrm{r}_{1}+\mathrm{r}_{2}-6 \mathrm{r}_{1} r_{2}\right)}{12 \mathrm{r}_{1} r_{2}+8 r_{1}+3 r_{2}} .
\end{aligned}
$$

These equations are valid if the principal axis of the normal mode of vibration lies along the long molecular axis, which can be assumed in the good approximation for the intramolecular vibration corresponding to the $\mathrm{C} \equiv \mathrm{N}$ stretch. In the other cases (i.e. $\mathrm{a} \neq \mathrm{b} \neq 0$ ) the more complicated calculations are needed and they are reported in details in reference [24].

Mostly pure liquid crystals had been investigated by using the RS spectroscopy, but some examples of the study for liquid crystals doped with guest molecules can also be found $[23,25,26]$.

\section{Conclusions}

Some examples of the utilization of various optical spectroscopy methods for investigations of thermotropic low molecular and polymeric liquid crystals as well as guest-host mixtures have been presented. It has been shown, that using these methods one can obtain information about both long-range orientational order and some direction-dependent molecular properties of the compounds investigated.

Comparing the values of the order parameters obtained by using various methods (see e.g. Fig. 3) it is seen, that some discrepancies occur. There can be many reasons of such differences. The most important one seems to be connected with the assumptions made in order to obtain the appropriate formula allowing to calculate $\mathrm{S}$ and $\mathrm{D}$ or $\left\langle\mathrm{P}_{2}\right\rangle$ and $\left\langle\mathrm{P}_{4}\right\rangle$. The way of the sample macroscopic orientation (magnetic or electric field, mechanical forces [1]) may also play some role. At any rate, all the methods described here allow to ascertain and to compare the orienting ability of various liquid crystals under investigation, which is a very important information from the technological point of view, especially in the case of novel synthesized substances.

\section{Acknowledgements}

This work was supported by Poznan University Research Project No TB 64-001/2001-DS.

\section{References}

[1] Liquid Crystals, H. Stegemeyer, ed., Steinkopff Darmstadt, Springer New York, 1994.

[2] J. Michl and E. W. Thulstrup, Spectroscopy with Polarized Light, VCH Publishers, Inc., New York, 1986.

[3] Liquid Crystals and Plastic Crystals, vol. 2, G. W. Gray and P. A. Winsor, eds., Academic Press, New York, 1979.

[4] The Molecular Physics of Liquid Crystals, G. R. Luckhurst and G. W. Gray, eds., Academic Press, New York, 1979.

[5] W. Maier and G. Englert, Z. Elektrochem., 64, 689 (1960).

[6] T. S. Hanson, Z. Naturforsch., 24a, 866 (1969).

[7] W. Haase, O. Trinquet, U. Quotschalla and J. K. Foitzik, Mol. Cryst. Liq. Cryst., 148, 15 (1987).

[8] A. V. Ivashchenko and G. V. Rumyantsev, Mol. Cryst. Liq. Cryst., 150, 1 (1987).

[9] D. Bauman, Mol. Cryst. Liq. Cryst., 159, 197 (1988).

[10] H.-G. Kuball, R. Memmer, A. Strauss, M. Junge, G. Scherowsky and A. Schönhofer, Liq. Cryst., 5, 969 (1989).

[11] E. Wolarz, H. Moryson and D. Bauman, Displays, 13, 171 (1992).

[12] W. E. Haas, Mol. Cryst. Liq. Cryst., 94, 1 (1983).

[13] D. Bauman and H.-G. Kuball, Chem. Phys., 176, 221 (1993).

[14] D. Bauman, C. Killet, S. E. Boiadjev, D. A. Lightner, A. Schönhofer and H.-G. Kuball, J. Phys. Chem., 100, 11546 (1996).

[15] D. Bauman, H. Schulze and H.-G. Kuball, Liq. Cryst., 27, 1357 (2000).

[16] F. C. Saunders, L. Wright and M. G. Clark, in: Liquid Crystals and Ordered Fluids, vol. 4, A. C. Griffin and J.F. Johnson, eds., Plenum Press, New York, London, 1984.

[17] D. Bauman and H. Moryson, J. Mol. Structure, 404, 113 (1997).

[18] E. Sackmann and D. Rehm, Chem. Phys. Lett., 4, 537 (1970).

[19] C. Zannoni, Mol. Phys., 38, 1813 (1979).

[20] E. Wolarz and D. Bauman, Liq. Cryst., 19, 221 (1995).

[21] L. L. Chapoy, D. B. Du Pré and E. T. Samulski, in: Liquid Crystals and Ordered Fluids, vol. 3, Plenum Press, New York, London, 1978.

[22] E. Wolarz, Z. Naturforsch., 47a, 807 (1992).

[23] M. Kozielski, D. Bauman, M. Drozdowski and Z. Salamon, Mol. Cryst. Liq. Cryst., 142, 1 (1987).

[24] S. Jen, N. A. Clark, P. S. Pershan and E. B. Priestley, J. Chem. Phys., 6, 4635 (1977).

[25] Y. Nakajima, H. Yoshida, S. Kobinata and S. Maeda, J. Phys. Soc. Jap., 49, 1140 (1980).

[26] D. Wróbel, Biophys. Chem., 26, 91 (1987).

\section{Author}

\section{Danuta Bauman}

Poznan University of Technology

Faculty of Technical Physics

ul. Nieszawska 13a

60-965 Poznan, Poland

Tel. +48 (61) 6652325

E-Mail: bauman@phys.put.poznan.pl 